\title{
When Like Destabilizes Like: Inverted Solvent Effects in Apolar Nanoparticle Dispersions
}

Debora Monego,,$\ddagger$ Thomas Kister, Nicholas Kirkwood, ${ }^{\S}$ David Doblas, $₫$ Paul Mulvaney, ${ }^{\S}$ Tobias Kraus, ${ }^{\uparrow, \|}$ and Asaph Widmer-Cooper, $, \dagger, \ddagger$

$\dagger A R C$ Centre of Excellence in Exciton Science, School of Chemistry, The University of Sydney, NSW 2006, Australia

$\ddagger$ The University of Sydney Nano Institute, The University of Sydney, NSW 2006, Australia IINM - Leibniz Institute for New Materials, Campus D2 2, 66123 Saarbrücken, Germany $\S A R C$ Centre of Excellence in Exciton Science, School of Chemistry, University of Melbourne, Parkville, Victoria 3010, Australia

||Colloid and Interface Chemistry, Saarland University, Campus D2 2, 66123 Saarbrücken, Germany

E-mail: asaph.widmer-cooper@sydney.edu.au

Figure 1 shows the scattering curves of AuNPs and the structure factor contributions during a cooling cycle. The development of the first peak in the structure factor indicates the agglomeration of the nanoparticles. 

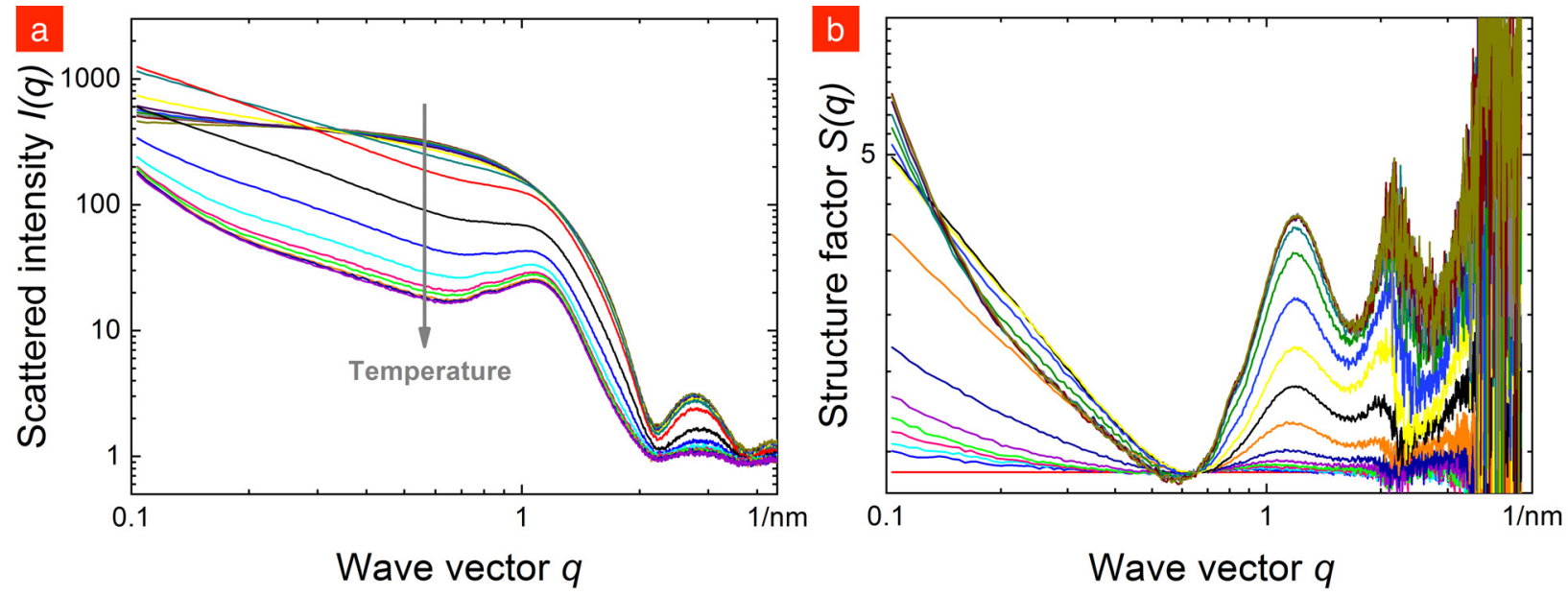

Figure 1: Temperature-dependent scattering of $4 \mathrm{~nm}$ Au nanoparticles. a) Raw data. b) The calculated structure factors. The peaks increase upon cooling.

The trend observed for the agglomeration temperature when changing the length of the alkane in which they are dispersed is the same for particles with a larger core diameter: $\mathrm{Au}$ particles of $7.5 \mathrm{~nm}$ in diameter coated with hexadecanethiol ligands (Figure 2).

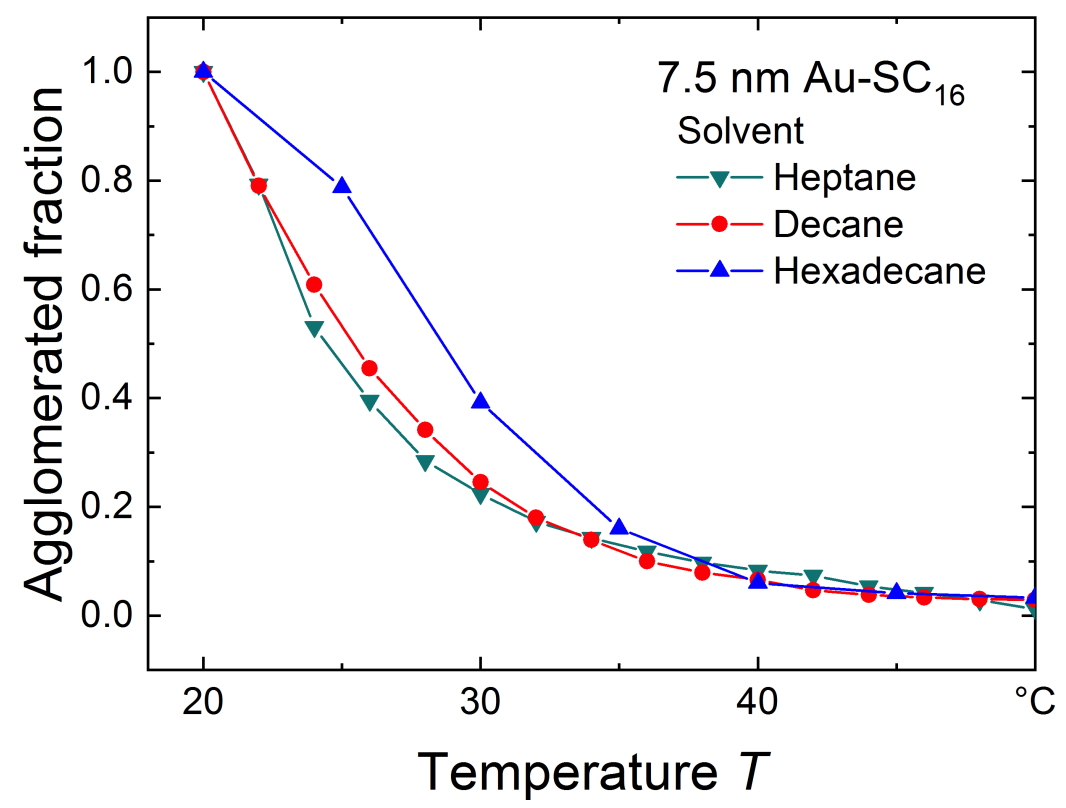

Figure 2: Fraction of agglomerated $7.5 \mathrm{~nm} \mathrm{Au}-\mathrm{SC}_{16}$ particles as determined by in situ small-angle X-ray scattering. 
Table 1 shows the solubility parameters, from both Hildebrand and Hansen theories, for the solvents studied here.

Table 1: Hildebrand and Hansen solubility parameters for the solvents studied here and the ligand-solvent interaction Flory parameter $(\chi)$, calculated as per Equation 1. Solubility parameters for the ligands were approximated by the ones of the unthiolated alkane.

\begin{tabular}{|c|c|c|c|c|c|c|c|}
\hline & Ligand & \multicolumn{5}{|c|}{ Solvent } & \multirow[b]{2}{*}{ Flory Parameter } \\
\hline & Hildebrand Parameter $\left(\mathrm{Pa}^{1 / 2}\right)^{1}$ & & Hildebrand Parameter $\left(\mathrm{Pa}^{1 / 2}\right)^{1}$ & \multicolumn{3}{|c|}{$\begin{array}{l}\text { Hansen Parameter }{ }^{1} \\
\text { dispersion dipolar } H_{\text {bond }}\end{array}$} & \\
\hline \multirow{4}{*}{$\mathrm{SC}_{16}$} & \multirow{4}{*}{16400} & Hexane & 14100 & 14100 & 0 & 0 & 0.62 \\
\hline & & Decane & 15800 & 15800 & 0 & 0 & 0.37 \\
\hline & & Hexadecane & 16400 & 16400 & 0 & 0 & 0.34 \\
\hline & & Cyclohexane & 16800 & 16800 & 0 & 200 & 0.35 \\
\hline \multirow{4}{*}{$\mathrm{SC}_{18}$} & \multirow{4}{*}{17100} & Hexane & 14100 & 14100 & 0 & 0 & 0.82 \\
\hline & & Decane & 15800 & 15800 & 0 & 0 & 0.47 \\
\hline & & Hexadecane & 16400 & 16400 & 0 & 0 & 0.40 \\
\hline & & Cyclohexane & 16800 & 16800 & 0 & 200 & 0.34 \\
\hline
\end{tabular}

Figure 3 shows snapshots of our MD simulations of CdSeNPs at temperatures around the experimental agglomeration temperature $T_{\text {agglo }}$ in alkane solvents with different chain lengths. Similarly to what is observed for AuNPs, the ligands go through a disorder-order transition when the temperature is decreased. This is responsible for changing the overall interaction between the ligand shells, switching the total interaction potential between nanoparticles from repulsive to attractive, as can be seen in Figure 5. 

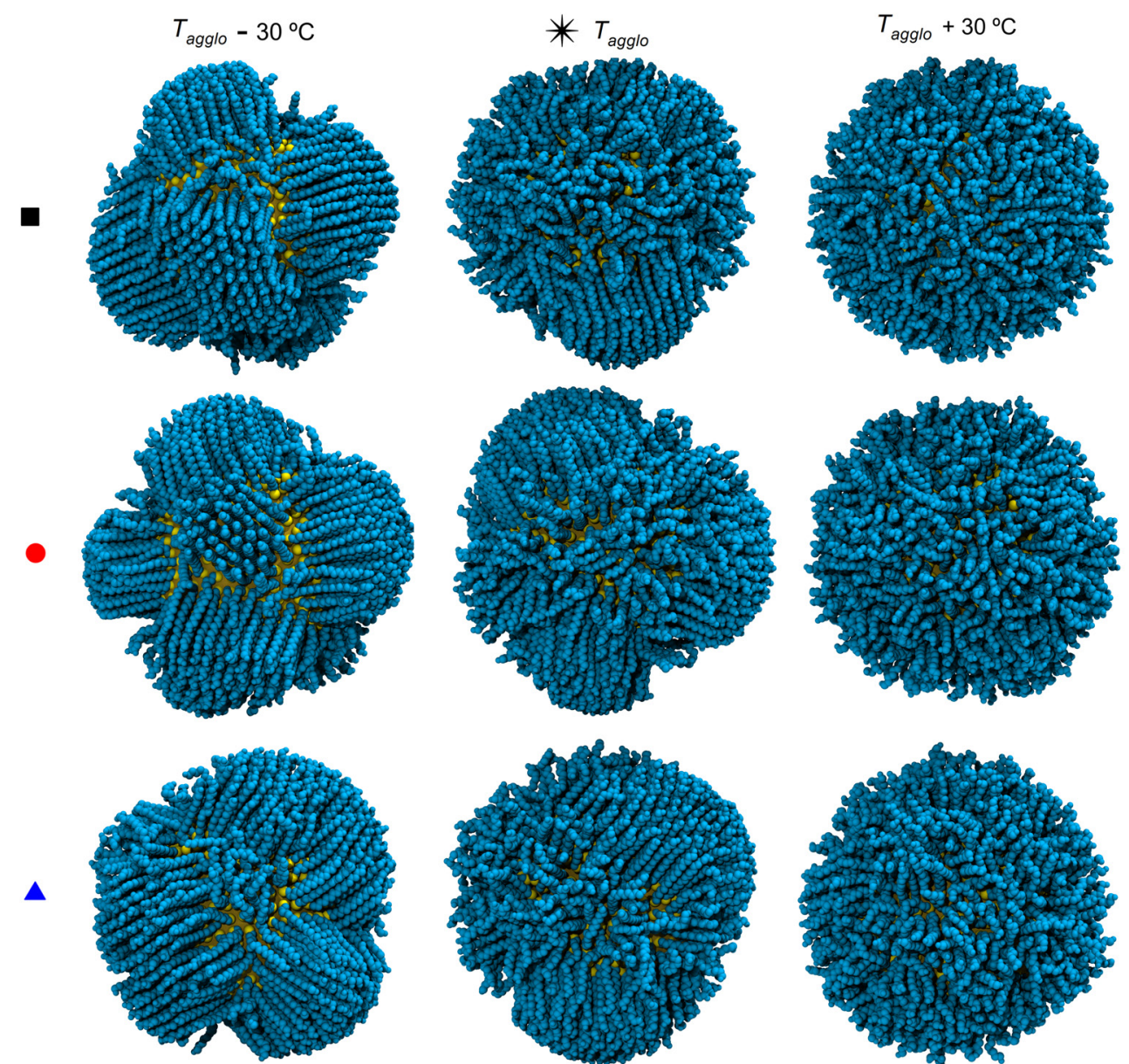

Figure 3: Simulation snapshots of $5.8 \mathrm{~nm} \mathrm{CdSe}-\mathrm{SC}_{18}$ particles at the experimental $T_{\text {agglo }} \pm$ $30^{\circ} \mathrm{C}$ in hexane (square), decane (circle), and hexadecane (triangle). Solvent molecules have been hidden for clarity.

Figure 4 shows snapshots and radial distribution functions for $4 \mathrm{~nm} \mathrm{Au}-\mathrm{SC}_{16}$ particles in cyclohexane. The ligands order when the temperature is decreased in a similar way to when the particles are dispersed in linear alkanes. 
a

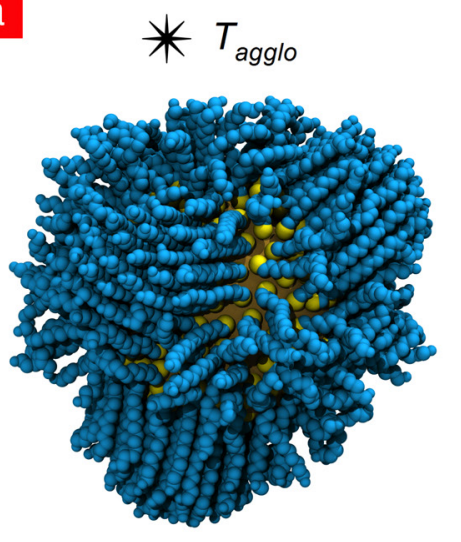

$$
T_{\text {agglo }}+30^{\circ} \mathrm{C}
$$

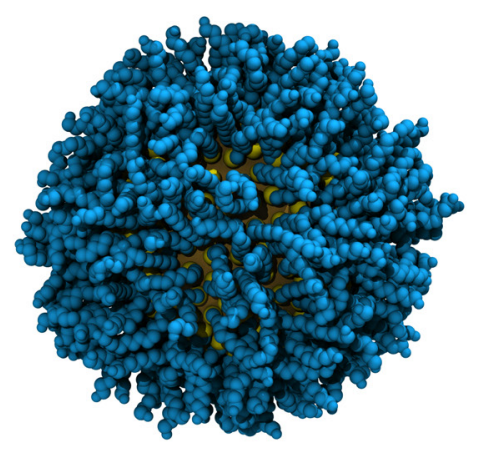

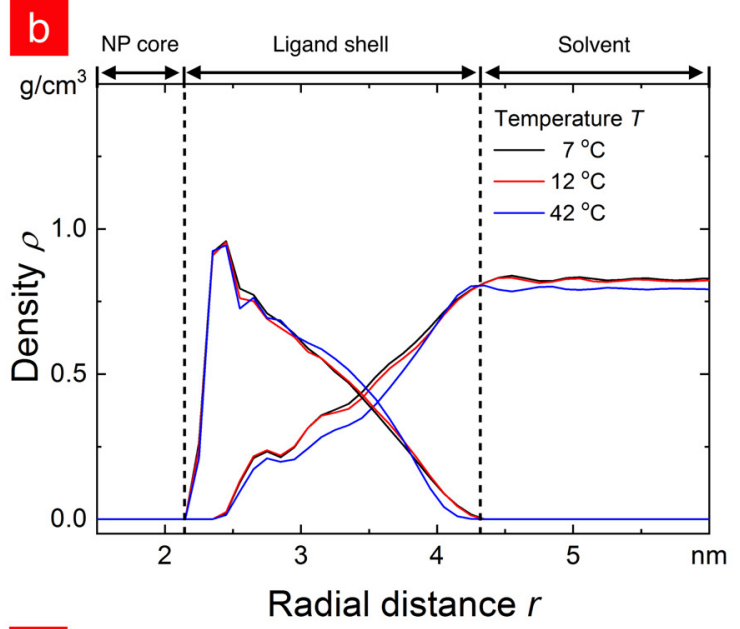

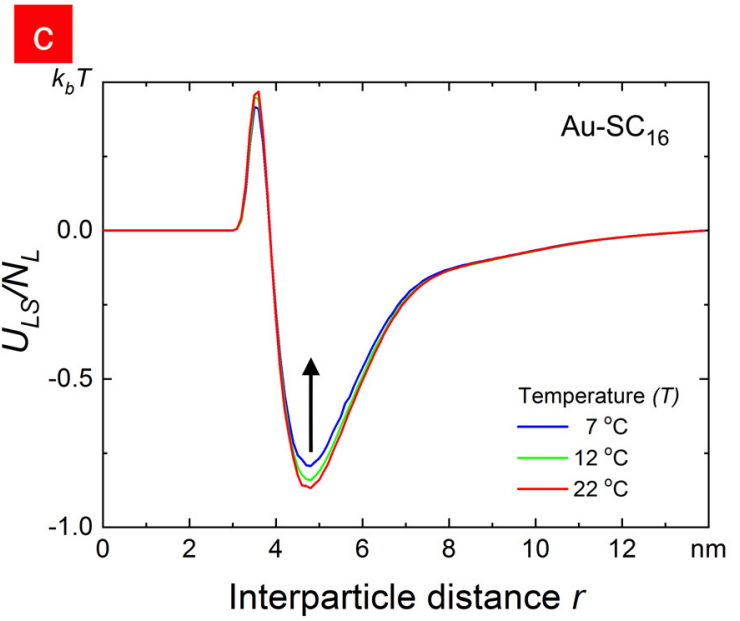

Figure 4: $4 \mathrm{~nm} \mathrm{Au}-\mathrm{SC}_{16}$ particles in cyclohexane: (a) Simulation snapshots at the experimental $T_{\text {agglo }}$ and $T_{\text {agglo }}+30^{\circ} \mathrm{C}$. Solvent molecules have been hidden for clarity. (b) Radial density distributions for the ligand and solvent molecules as a function of the distance $r$ from the center of the nanoparticle core at (red) and above (blue) $T_{\text {agglo }}$. (c) Contribution to the total ligand-solvent interaction energy as a function of distance between pairs of interacting $\mathrm{CH}_{\mathrm{x}}$ groups. Energies are normalized by the number of ligand molecules on the nanoparticle $N_{L}$. 

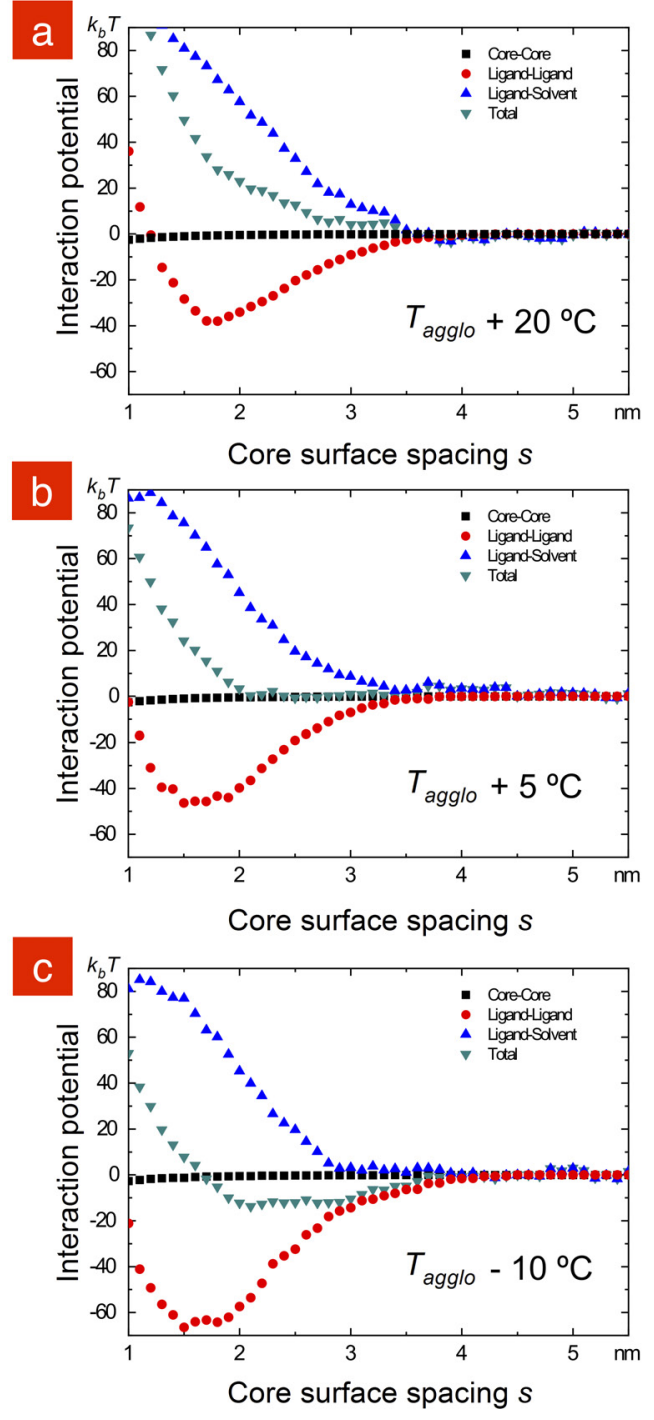

Figure 5: Components of the Potential of Mean Force (PMF) obtained from constrained molecular dynamics simulations for pairs of $4 \mathrm{~nm} \mathrm{Au}-\mathrm{SC}_{16}$ particles in hexane at temperatures around the experimental $T_{\text {agglo }}$. The total potential is green, the ligand-ligand contribution is red, the ligand-solvent contribution is blue, and the core-core contribution is black.

Figure 6 shows the total number of pair interactions between ligand $\mathrm{CH}_{\mathrm{x}}$ groups as a function of the separation between them, the solvent and the temperature. The temperature at which the ligands order $\left(T_{\text {order }}\right)$ differs between solvents, but the average structure of the ligand shell is virtually the same in all solvents when the temperature is expressed relative to $T_{\text {order }}$. Radial density distributions for the ligand and solvent molecules surrounding the 
particles are shown in Figure 7.
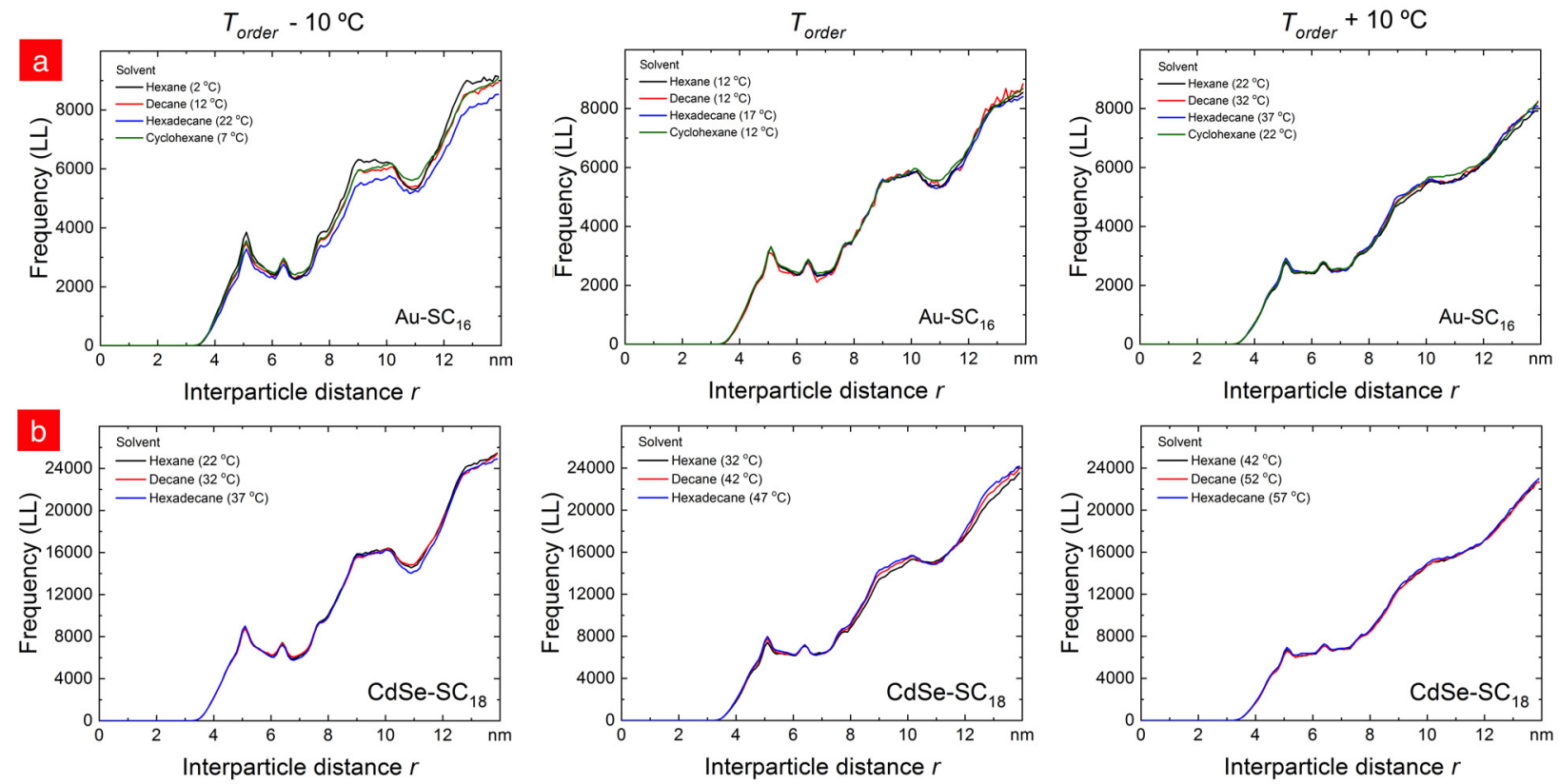

Figure 6: The number of ligand-ligand $\mathrm{CH}_{\mathrm{x}}-\mathrm{CH}_{\mathrm{x}}$ interactions as a function of the distance between them for (a) $4 \mathrm{~nm} \mathrm{Au}-\mathrm{SC}_{16}$ and (b) $5.8 \mathrm{~nm} \mathrm{CdSe-} \mathrm{SC}_{18}$ particles shows that the ligands order similarly in all solvents for the same temperature relative to the ordering transition. 

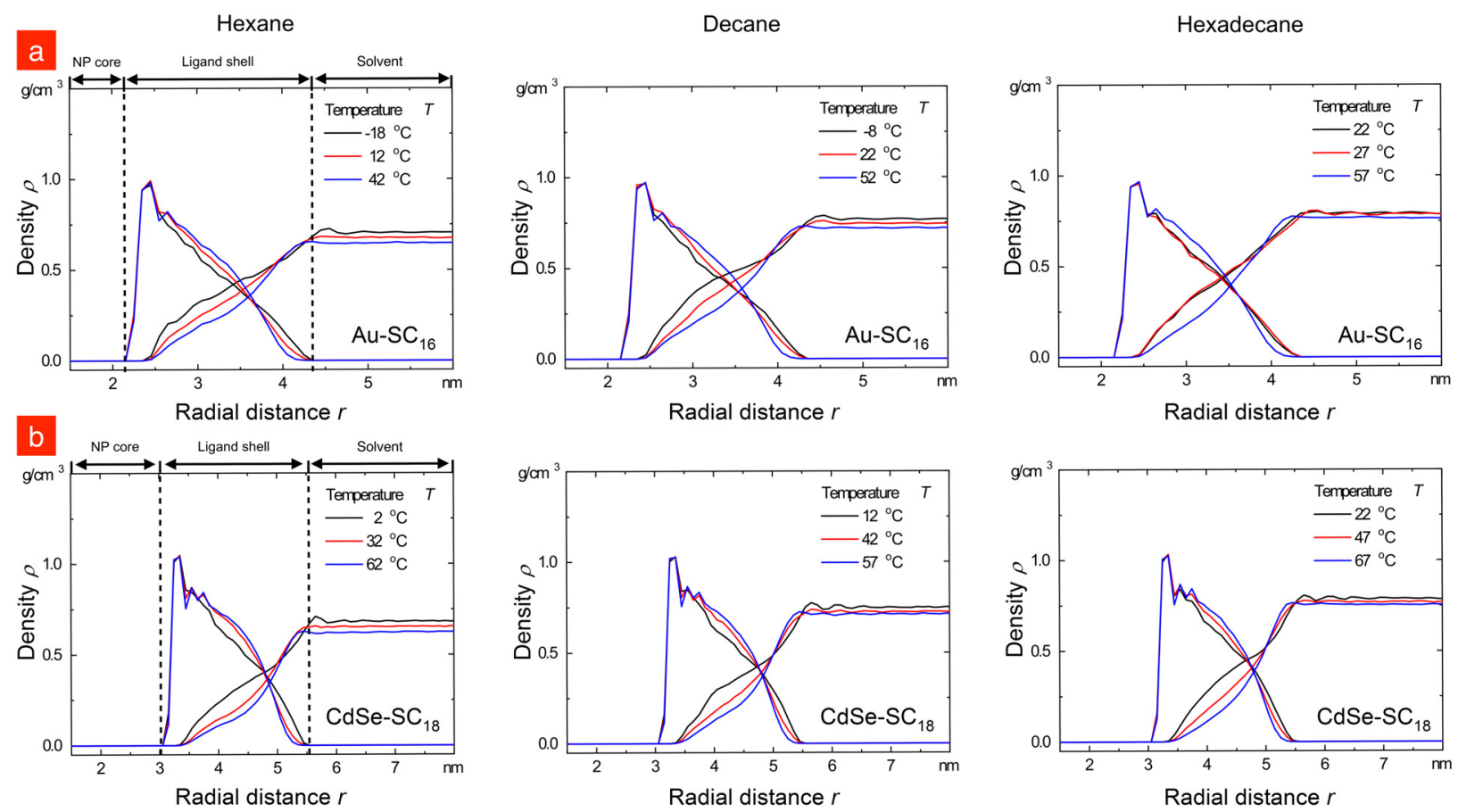

Figure 7: Radial density distributions for the ligand and solvent molecules for Au and CdSe $\mathrm{NP}$ in different alkane solvents and temperatures, plotted as a function of the distance $r$ from the center of the nanoparticle core. The red lines indicate the density profile at the ligand ordering temperature $T_{\text {order }}$. In all cases, the radially averaged solvent density within the ligand shell increases upon cooling.

Figure 8 shows the contribution to the ligand-solvent interaction energy as a function of the distance between $\mathrm{CH}_{\mathrm{x}}$ groups. Energies are normalized by the number of ligands on the nanoparticle: 280 ligands for AuNP, and 580 ligands for CdSeNP. 

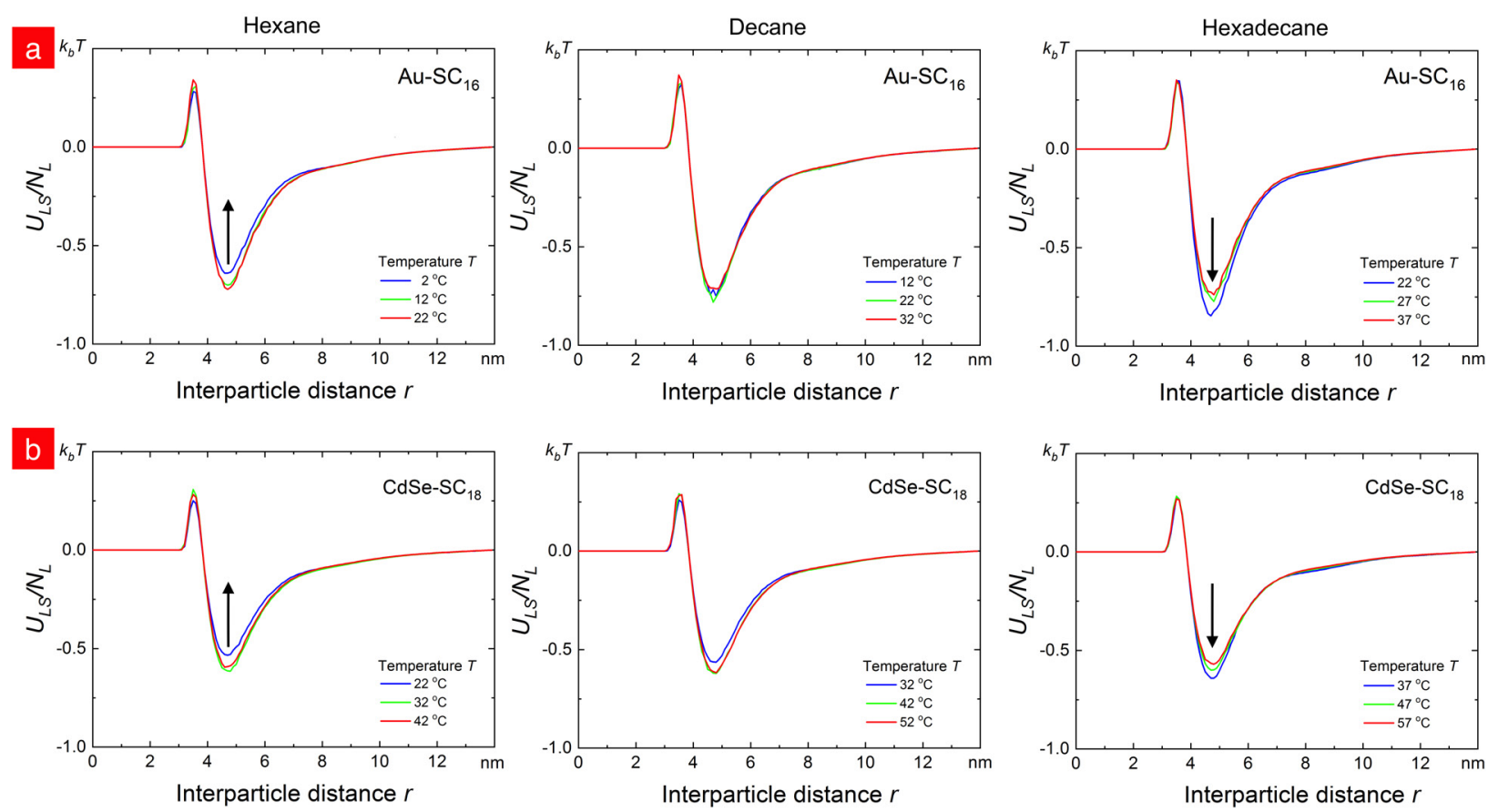

Figure 8: Contribution to the ligand-solvent interaction energy as a function of distance between pairs of interacting $\mathrm{CH}_{\mathrm{x}}$ groups for (a) $4 \mathrm{~nm} \mathrm{Au}-\mathrm{SC}_{16}$ and (b) $5.8 \mathrm{~nm} \mathrm{CdSe}-\mathrm{SC}_{18}$ particles in different solvents. For longer alkanes, there is an increase in the number of ligand and solvent atoms that are close to one another upon cooling. All energies are normalized by the number of ligand molecules on the nanoparticle $N_{L}$.

The average dihedral angle of the solvent molecules (Figure 9 for CdSeNPs) was calculated separately for two regions of the simulation cell: (i) within the spherical ligand shell, i.e. the region where the radially averaged ligand density is nonzero; and (ii) a region sufficiently far from the nanoparticle that the solvent molecules behave as bulk solvent. 


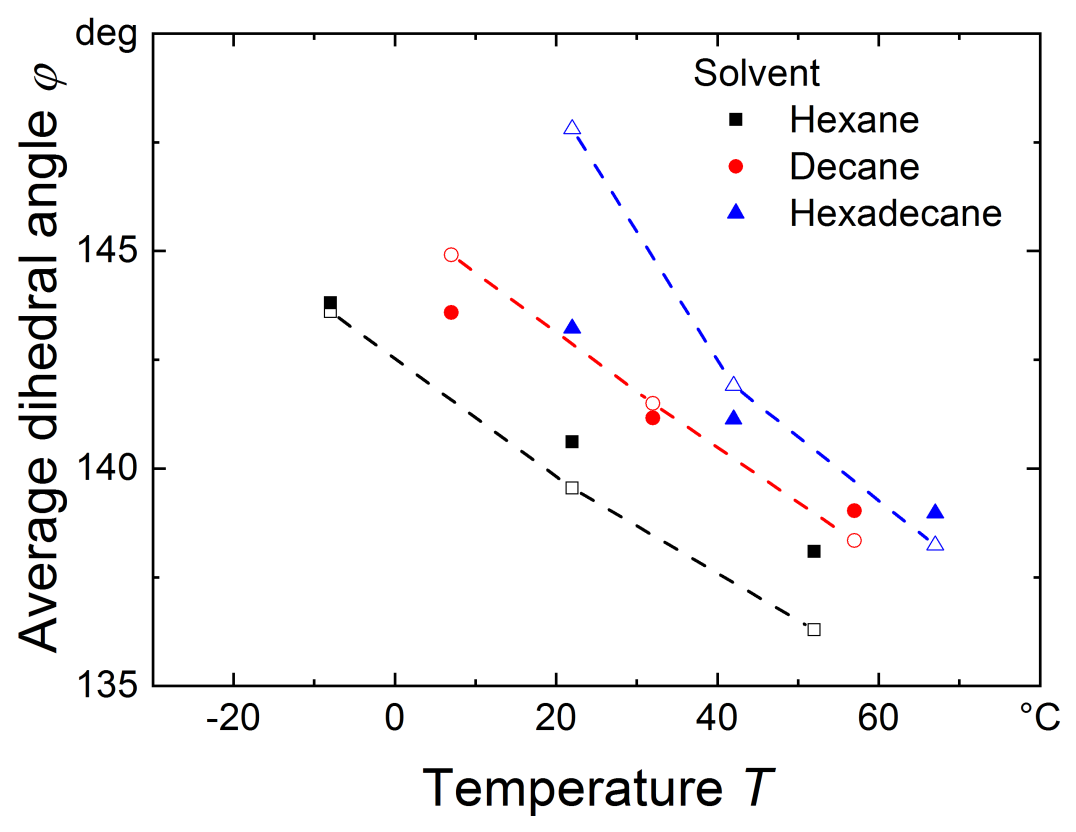

Figure 9: Average dihedral angle of solvent molecules that are in the bulk region (closed symbols) and within the 1-octadecanethiol ligand shell (open symbols) covering $5.8 \mathrm{~nm}$ CdSeNP.

Table 2: Parameters used for analytical calculations of the entropies of mixing using equations 1 and 2 .

\begin{tabular}{ll}
\hline \multicolumn{1}{c}{ parameter } & \multicolumn{1}{c}{ value } \\
\hline \hline ligand surface coverage & 5.5 ligands nm \\
ligand length $L$ for hexadecanethiol & $2.28 \mathrm{~nm}$ \\
ligand length $L$ for octadecanethiol & $2.54 \mathrm{~nm}$ \\
volume ligand molecule $\nu_{L}$ for hexadecanethiol & $0.550 \mathrm{~nm}^{3}$ \\
volume ligand molecule $\nu_{L}$ for octadecanethiol & $0.616 \mathrm{~nm}^{3}$ \\
volume solvent molecule $\nu_{S}$ for hexane & $0.215 \mathrm{~nm}^{3}$ \\
volume solvent molecule $\nu_{S}$ for decane & $0.324 \mathrm{~nm}^{3}$ \\
volume solvent molecule $\nu_{S}$ for hexadecane & $0.487 \mathrm{~nm}^{3}$ \\
\hline \hline
\end{tabular}

\section{References}

1. Brandrup, J.; Immergut, E. H.; Grulke, E. A. Polymer Handbook; Wiley-Interscience: New York, 1999; p 2250. 